\title{
Inferring Transcription Factors and microRNAs Associated with Elevated Expression of the Oncogenic B-Cell Lymphoma 11A in Triple Negative Breast Cancer
}

\section{Nouf N. Laqtom, PhD, Khloud M. Algothmi, PhD, Amani H. Bakhribah, BSc}

Department of Biological Sciences, Division of Genomics and Biotechnology, Faculty of Science, King Abdulaziz University, Jeddah, Saudi Arabia

\section{Correspondence}

Dr. Nouf N. Laqtom

P.O. Box 42671, Jeddah 21551

e.M: nlaqtom@kau.edu.sa

Submission: 24 Apr. 2016

Accepted: 5 Jun. 2016

\section{Citation}

Laqtom NN, Algothmi KM, Bakhribah AH. Inferring transcription factors and microRNAs associated with elevated expression of the Oncogenic B-Cell Lymphoma 11A in Triple Negative Breast Cancer. JKAU Med Sci 2016; 23

(3): 9-21. DOl: 10.4197/Med. 23.3.2

\begin{abstract}
B-cell lymphoma 11A, a transcriptional repressor, is highly expressed in triple negative breast cancer. The in vitro studies and animal models provide initial evidence suggesting that the knockdown of B-cell lymphoma 11A has a therapeutic effect on breast cancer. Defining the regulators driving the high expression of B-cell lymphoma $11 \mathrm{~A}$ is important to understand its cancer-related functions. Among these regulators, transcription factors and microRNAs are critical for gene expression and associated with expression perturbations. Firstly, we identified the transcription factors that potentially interact with B-cell lymphoma 11A promoter. Based on bioinformatics prediction and multiple Omics datasets, two upregulated transcriptional activators Zinc Finger BED-Type Containing 4 and E2F Transcription Factor 1 in triple negative breast cancer were found to have seven sites within B-cell lymphoma 11A promoter. Secondly, we aimed to determine a putative set of microRNA that can mediate the post-transcriptional repression of B-cell lymphoma 11A. miR-513a-5p, miR-139-5p, miR1179 , miR-140-5p, and miR-542-3p, harboring at least one site of interaction with B-cell lymphoma 11A 3' untranslated region, were found inhibited in triple negative breast cancer. Taken together, the combinatorial regulation by transcription factors and microRNAs provide valuable information for further investigation on controlling the expression level of B-cell lymphoma 11A in triple negative breast cancer.
\end{abstract}

\section{Keywords}

B-cell lymphoma 11A; Breast cancer; Transcription factors; microRNA; Omics; Bioinformatics 


\section{Introduction}

B reast cancer is the second major cause of death in economically developed and less developed countries. Nearly 1.67 million new cases of breast cancer were diagnosed around the world in 2012 and about 522,000 died of this cancer ${ }^{[1]}$. Breast cancer is a heterogeneous disease and each of its subtypes can be associated with a unique set of biomarkers. B-cell lymphoma 11A (BCL11A), a multiple $\mathrm{C} 2 \mathrm{H} 2$ zinc finger containing transcription factor, was identified to be elevated in breast cancer tissues compared with normal tissues, particularly triple negative breast cancer stem and progenitor cells $s^{[2]}$. Triple negative breast cancer (TNBC) subtype accounts for 10-15\% of all breast cancers, however, it is associated with a higher recurrence and poorer survival rates when compared with other subtypes. Disruption of BCL11A expression in TNBC cell lines and in xenograft mouse models significantly reduced the cancer development and progression. Consistent with this, the deletion of BCL11A in a DMBA-induced tumor mice model substantially decreased tumor formation as well as in p53-null mice model for cancer. The transcription factor, BCL11A, appears to be a novel oncogenic target for the treatment of TNBC breast cancer ${ }^{[2]}$. Moreover, the high expression of BCL11A appears to have a critical role in other aggressive cancers such as $B$ cell chronic lymphocytic leukemia $(C L L)^{[3]}$.

Though the role of $B C L 11 A$ in the tumorigenesis has been studied, its transcriptional regulation remains unknown. However, its transcriptional regulation of $\mathrm{BCL11A}$ has been investigated in other biological contexts such as the regulation of erythropoiesis and fetal hemoglobin level ${ }^{[4]}$. Kruppel Like Factor 1 (KLF1) is a direct transcriptional activator of BCL11A gene in erythroid progenitor cells $s^{[5]}$. Besides transcription factors, microRNAs (miRNAs) are critical regulators for the post-transcriptional and/or translational level of gene expression. Recent studies reported significant evidence that the deregulated miRNAs regulate the expression of their target genes in cancer ${ }^{[6]}$. The main role of miRNAs is to inhibit protein expression through interaction with seed complement sites to the $3 \rrbracket$ untranslated region ( $3^{\prime} \cup T R$ ) of its target messenger RNA (mRNAs), and thereby negatively influence mRNA translation $^{[7]}$. There is no work that has identified miRNAs able to regulate the expression of BCL11A protein in breast cancer cells.

In this paper we address two central questions concerning the transcriptional and post-transcriptional regulation of $B C L 11 \mathrm{~A}$ in breast cancer cells using a combinatorial method of bioinformatics and omic analysis. (1) Whether high expressed transcription factors with the potential to enhance transcription through direct interaction with BCL11A promoter region were present in breast cancer tissues compared with the controls, and (2) whether a decrease in the expression of miRNAs can result in the high expression of BCL11A in breast cancer versus the control cells.

\section{Methods}

\section{The Sequence of BCL11A Promoters}

The promoter sequences of mouse and human BCL11A were obtained using the GenBank nucleotide sequence database (National Center for Biotechnology Information, Bethesda, MA USA).

\section{MatInspector Prediction Search}

MatInspector prediction search (Genomatix $\mathrm{GmbH}$, Munich, Germany) for transcription factor binding sites (TFBSs) is based on a number of parameters including the core and matrix similarities, which are calculated in MatInspector. The core similarity was set to 1 and $>0.95$ for the matrix, whereas the optimized matrix threshold was left at the default leve[ ${ }^{[8]}$.

\section{TumourProfile Database}

TumourProfile database (Peking University Center for Human Disease Genomics, Beijing, China) (http:// tumour.bjmu.edu.cn/) is a database on gene expression profile (GEP) across multiple datasets, mainly microarray transcriptional profiling, of human normal, non-cancerous, cancerous tissues, and cancer cell lines. The $P$ values and Pearson's correlation coefficient $(r)$ between tumour profiles were calculated in the database to measure the strength of the correlation between two variables.

\section{BioXpress v1.0}

BioXpress (https://hive.biochemistry.gwu.edu/tools/ bioxpress/) is a database for curated gene expression in normal and cancer tissues. Based on RNA-seq data provided by The Cancer Genome Atlas, International Cancer Genome Consortium, Expression Atlas and publications, the expression level of genes were calculated $^{[9]}$. 


\section{Oncomine ${ }^{\mathrm{TM}}$}

Oncomine $^{\mathrm{TM}}$ (Thermo Fisher Scientific Inc., Waltham, MA USA) (www.oncomine.org) is a curated database containing a large number of cancer transcriptome profiles. The analysis engine is used to measure the differential expression comparing diverse cancer tissues with respective normal tissues as well as cancer subtypes $^{[10]}$.

\section{Human Protein Atlas}

Human Protein Atlas (The Knut and Alice Wallenberg Foundation, Stockholm, Sweden) (www.proteinatlas. org/) is a database for human protein expression patterns in normal and disease tissues using a range of experimental procedures, from the classical experiments such as Elisa, Western blot and immunohistochemistry to antibody-based proteomics approaches $^{[11]}$.

\section{Triple-Negative Breast Cancer Database}

Triple-Negative Breast Cancer Database (Rajiv Gandhi Centre for Biotechnology, Kerala, India) (http://210.212.254.116/tnbcdb/) is a curated database of the expression profile of miRNA, mRNA, protein and phosphoprotein levels. Changes in expression are measured in comparison with the normal tissues or other subtypes of breast cancers ${ }^{[12]}$.

\section{TargetScan}

TargetScan (Whitehead Institute for Biomedical Research, Cambridge, MA USA), (www.targetscan. org/) is in silico miRNA target prediction; an algorithm was developed to predict miRNA sites based on the rules and patterns of interaction between mRNAs and miRNAs.

\section{Results}

\section{Identification of Potential Transcription Factors Related to the High Expression of BCL11A in Breast Cancers}

The $\mathrm{BCL} 11 \mathrm{~A}$ promoter region was retrieved from National Centerfor Biotechnology Information database (June, 2016). The length of retrieved promoter is $600 \mathrm{bp}$ (from $500 \mathrm{bp}$ upstream to $100 \mathrm{bp}$ downstream of the transcription start site (TSS)). In order to identify the transcription factor binding sites of $\mathrm{BCL} 11 \mathrm{~A}$ promoter the sequence was uploaded in Matlnspector with core and matrix similarity cut-offs 1 and 0.95 , respectively. At least 22 transcription factor families were predicted to occupy at least two interaction sites in BCL11A promoter (Table 1). These families include 33 different transcription factors. Next, we assessed which of these 33 transcription factors can be responsible for the high expression of BCL11A in breast cancer cells. Therefore, each transcription factor was uploaded in three different databases (BioXpress v1.0, Oncomine ${ }^{\mathrm{TM}}$, and Human Protein Atlas (June, 2016)) to determine their expression levels in normal breast tissue compared to cancer tissues. Each transcription activator or repressor has a consistent upregulation or downregulation in at least two databases included in our results (Table 2). There are 5 upregulated transcriptional activators (Zinc Finger BED-Type Containing 4 (ZBED4); WT1, Zinc Finger and BTB Domain Containing 7B (ZBTB7B); Zinc Finger Protein 35 (ZNF35); E2F1) and 3 downregulated repressors (Kruppel Like Factor 6 (KLF6); Zinc Finger Protein 300 (ZNF300); Zinc Finger Protein 219 (ZNF219)) in breast cancers. Increased transcriptional activators and inhibited repressors may contribute to the increased expression of BCL11 A in breast cancers.

\section{Identification of Potential Transcription Fac- tors Related to the High Expression of BCL11A in TNBC Compared to Non-TNBC}

In order to further assess the potential transcription factors involved in the increased BCL11A in triple negative breast cancer, we used the omics dataset from the Gene Expression Omnibus (GEO) database (June, 2016) to determine the differential expression of the transcription factors between triple negative and nontriple negative breast cancer. At least 3 transcriptional activators (ZBED4, ZNF35, E2F Transcription Factor 1 (E2F1)) showed a higher level in triple negative than its expression level in non-triple negative breast cancer (Table 3). However, no transcriptional repressors displayed a further decreased level in triple negative breast cancer. The increased level of the transcriptional activators ZBED4, ZNF35, E2F1, may contribute to the higher $\mathrm{BCL} 11 \mathrm{~A}$ in triple negative breast cancer compared with other breast cancer subtypes.

\section{Correlation between BCL11A and Transcription Factor Expression}

We then assessed whether the expression of BCL11A and its potential transcription factors (ZBED4, ZNF35, E2F1) are co-regulated. Therefore, Pearson's correlations were calculated in TumourProfile database. As shown 
Inferring Transcription Factors and microRNAs Associated with Elevated Expression of the Oncogenic B-Cell Lymphoma 11A...

N.N. Laqtom et al.

Table 1. A list of predicted matrix families and their specific transcription factors. The Mallnspector software was used and its parameters set the core and matrix similarities to 1 and 0.95 . Each family with two or more binding sites was included. The table also includes the position and strand, where each transcription factor can bind to the sequence of BCL11A promoter.

\begin{tabular}{|c|c|c|c|c|c|c|}
\hline Matrix Family & Detailed Family Information & Matrix & Detailed Matrix Information & Location & Strand & Matrix Similarity \\
\hline BEDF & BED subclass of zinc-finger proteins & ZBED4 & $\begin{array}{l}\text { Zinc finger, BED-type containing } \\
\text { 4; GC-box binding sites }\end{array}$ & $\begin{array}{c}5-19 \\
34-48 \\
36-50 \\
138-152 \\
209-223 \\
193-207\end{array}$ & $\begin{array}{l}+ \\
- \\
- \\
- \\
- \\
-\end{array}$ & $\begin{array}{c}0.956 \\
0.96 \\
0.957 \\
0.958 \\
0.976 \\
0.96\end{array}$ \\
\hline CIZF & CAS interacting zinc finger protein & ZNF384 & Zinc finger protein 384 & $\begin{array}{l}246-256 \\
428-438 \\
507-517\end{array}$ & $\begin{array}{l}- \\
- \\
-\end{array}$ & $\begin{array}{c}0.965 \\
0.96 \\
0.961\end{array}$ \\
\hline \multirow[t]{2}{*}{ E2FF } & \multirow{2}{*}{$\begin{array}{l}\text { E2F-myc activator/cell cycle } \\
\text { regulator }\end{array}$} & E2F4 & $\begin{array}{l}\text { E2F transcription factor 4, } \\
\text { p107/p130-binding protein }\end{array}$ & $148-164$ & - & 0.976 \\
\hline & & E2F1 & E2F transcription factor 1 & $191-207$ & - & 0.988 \\
\hline \multirow{3}{*}{ EGRF } & \multirow{3}{*}{$\begin{array}{l}\text { EGR/nerve growth factor induced } \\
\text { protein C \& related factors }\end{array}$} & WT1 & Wilms Tumor Suppressor & $\begin{array}{c}4-22 \\
31-49\end{array}$ & $\begin{array}{l}+ \\
-\end{array}$ & $\begin{array}{c}0.984 \\
1\end{array}$ \\
\hline & & EGR2 & $\begin{array}{l}\text { Egr-2/Krox-20 early growth } \\
\text { response gene product }\end{array}$ & $\begin{array}{c}6-24 \\
29-47 \\
42-60\end{array}$ & $\begin{array}{l}+ \\
- \\
-\end{array}$ & $\begin{array}{l}0.965 \\
0.963 \\
0.981\end{array}$ \\
\hline & & ZBTB7B & $\begin{array}{l}\text { Zinc finger and BTB domain } \\
\text { containing } 7 \mathrm{~B})\end{array}$ & $152-170$ & - & 0.967 \\
\hline EVI1 & EVI1-myleoid transforming protein & PRDM16 & PR/SET domain 16 & $\begin{array}{l}432-448 \\
545-561\end{array}$ & $\begin{array}{l}- \\
-\end{array}$ & $\begin{array}{l}0.958 \\
0.963\end{array}$ \\
\hline \multirow{2}{*}{ HIFF } & \multirow{2}{*}{$\begin{array}{l}\text { Hypoxia inducible factor, } \\
\text { bHLH/PAS protein family }\end{array}$} & ARNT & $\begin{array}{l}\text { Aryl hydrocarbon receptor nuclear } \\
\text { translocator }\end{array}$ & $21-37$ & - & 0.985 \\
\hline & & HIF1A & $\begin{array}{c}\text { Hypoxia inducible factor } 1 \text { alpha } \\
\text { subunit }\end{array}$ & $384-400$ & - & 0.978 \\
\hline \multirow{3}{*}{ KLFS } & \multirow{3}{*}{ Kruppel like transcription factors } & KLF2 & Kruppel-like factor 2 (lung) (LKLF) & $\begin{array}{l}34-52 \\
47-65 \\
56-74\end{array}$ & $\begin{array}{l}- \\
- \\
-\end{array}$ & $\begin{array}{l}0.996 \\
0.996 \\
0.987\end{array}$ \\
\hline & & KLF3 & Kruppel-like factor 3 (basic) & $\begin{array}{l}136-154 \\
153-171 \\
207-225\end{array}$ & $\begin{array}{l}- \\
- \\
-\end{array}$ & $\begin{array}{c}0.994 \\
1 \\
0.995 \\
\end{array}$ \\
\hline & & KLF6 & $\begin{array}{l}\text { Core promoter-binding protein } \\
\text { (CPBP) with } 3 \text { Kruppel-type zinc }\end{array}$ & $203-221$ & - & 0.954 \\
\hline MAZF & Myc associated zinc fingers & MAZ & $\begin{array}{l}\text { Myc associated zinc finger protein } \\
\text { (MAZ) }\end{array}$ & $\begin{array}{l}144-156 \\
156-168\end{array}$ & $\begin{array}{l}- \\
-\end{array}$ & $\begin{array}{l}0.97 \\
0.97\end{array}$ \\
\hline MZF1 & Myeloid zinc finger 1 factors & MZF1 & Myeloid zinc finger protein MZF1 & $\begin{array}{c}11-21 \\
32-42 \\
142-152 \\
159-169 \\
207-217 \\
213-223 \\
289-299 \\
302-312\end{array}$ & $\begin{array}{l}+ \\
- \\
- \\
- \\
- \\
- \\
- \\
-\end{array}$ & $\begin{array}{l}0.996 \\
0.996 \\
0.995 \\
0.974 \\
0.986 \\
0.995 \\
1 \\
0.991\end{array}$ \\
\hline NDPK & Nucleoside diphosphate kinase & NME1 & $\begin{array}{l}\text { NME/NM23 nucleoside } \\
\text { diphosphate kinase } 1\end{array}$ & $\begin{array}{l}153-169 \\
207-223\end{array}$ & $\begin{array}{l}- \\
-\end{array}$ & $\begin{array}{l}0.957 \\
0.968\end{array}$ \\
\hline PLAG & Pleomorphic adenoma gene & PLAG1 & Pleomorphic adenoma gene 1 & $\begin{array}{c}8-30 \\
10-32 \\
21-43 \\
23-45 \\
125-147 \\
148-170 \\
167-189 \\
180-202 \\
196-218 \\
414-436\end{array}$ & $\begin{array}{l}+ \\
+ \\
- \\
- \\
- \\
- \\
+ \\
- \\
- \\
+\end{array}$ & $\begin{array}{c}0.959 \\
0.955 \\
0.955 \\
0.959 \\
1 \\
0.967 \\
0.967 \\
0.967 \\
1 \\
1\end{array}$ \\
\hline
\end{tabular}


Inferring Transcription Factors and microRNAs Associated with Elevated Expression of the Oncogenic B-Cell Lymphoma 11A...

N.N. Laqtom et al.

Table 1. (Continuation) A list of predicted matrix families and their specific transcription factors. The Mallnspector software was used and its parameters set the core and matrix similarities to 1 and 0.95. Each family with two or more binding sites was included. The table also includes the position and strand, where each transcription factor can bind to the sequence of BCL11A promoter.

\begin{tabular}{|c|c|c|c|c|c|c|}
\hline Matrix Family & Detailed Family Information & Matrix & $\begin{array}{l}\text { Detailed Matrix } \\
\text { Information }\end{array}$ & Location & Strand & $\begin{array}{c}\text { Matrix } \\
\text { similarity }\end{array}$ \\
\hline PURA & $\begin{array}{l}\text { Pur-alpha binds both single- } \\
\text { stranded and douple-stranded DNA } \\
\text { in a sequence-specific manner }\end{array}$ & PURA & $\begin{array}{l}\text { Purine-rich element binding } \\
\text { protein } \mathrm{A}\end{array}$ & $\begin{array}{l}153-165 \\
207-219 \\
293-305\end{array}$ & $\begin{array}{l}- \\
- \\
-\end{array}$ & $\begin{array}{l}0.961 \\
0.984 \\
0.963\end{array}$ \\
\hline SAL2 & Spalt-like transcription factor 2 & SALL2 & $\begin{array}{l}\text { Zinc finger protein Spalt-2, sal- } \\
\text { like 2, p150(sal2) }\end{array}$ & $\begin{array}{l}39-49 \\
52-62\end{array}$ & - & $\begin{array}{l}0.963 \\
0.969\end{array}$ \\
\hline SIX3 & Sine oculis homeobox homolog 3 & SIX3 & $\begin{array}{l}\text { SIX3 / SIXdomain (SD) and } \\
\text { Homeodomain (HD) } \\
\text { transcription factor }\end{array}$ & $\begin{array}{l}564-584 \\
575-595\end{array}$ & $\begin{array}{l}+ \\
+\end{array}$ & $\begin{array}{l}0.955 \\
0.961\end{array}$ \\
\hline SMAD & $\begin{array}{l}\text { Vertebrate SMAD family of } \\
\text { transcription factors }\end{array}$ & SMAD3 & $\begin{array}{l}\text { Smad3 transcription factor } \\
\text { involved in TGF-beta signaling }\end{array}$ & $\begin{array}{l}175-185 \\
354-364\end{array}$ & $\begin{array}{l}+ \\
-\end{array}$ & $\begin{array}{c}0.98 \\
1\end{array}$ \\
\hline SP1F & GC-Box factors SP1/GC & SP1 & $\begin{array}{l}\text { Stimulating protein } 1 \text {, } \\
\text { ubiquitous zinc finger } \\
\text { transcription factor }\end{array}$ & $\begin{array}{l}150-166 \\
193-209\end{array}$ & - & $\begin{array}{l}0.969 \\
0.997\end{array}$ \\
\hline & Activator/repressor binding to & YY2 & Transcription factor yin yang 2 & $422-444$ & + & 0.953 \\
\hline YYIF & transcription initiation site & YY1 & Yin and Yang 1 repressor sites & $558-580$ & + & 0.966 \\
\hline \multirow{5}{*}{ ZF02 } & \multirow{5}{*}{$\begin{array}{l}\mathrm{C} 2 \mathrm{H} 2 \text { zinc finger transcription } \\
\text { factors } 2\end{array}$} & ZNF148 & Zinc finger protein 148 & $\begin{array}{c}2-24 \\
204-226 \\
29-51\end{array}$ & $\begin{array}{l}- \\
+ \\
+\end{array}$ & $\begin{array}{l}0.959 \\
0.966 \\
0.955\end{array}$ \\
\hline & & ZKSCAN3 & $\begin{array}{l}\text { Zinc finger with KRAB and } \\
\text { SCAN domains } 3\end{array}$ & $\begin{array}{c}32-54 \\
35-57 \\
128-150\end{array}$ & $\begin{array}{l}+ \\
+ \\
+\end{array}$ & $\begin{array}{c}0.973 \\
1 \\
0.953\end{array}$ \\
\hline & & ZNF300 & $\begin{array}{l}\text { KRAB-containing zinc finger } \\
\text { protein } 300\end{array}$ & $\begin{array}{c}51-73 \\
126-148 \\
152-174 \\
134-156 \\
195-217 \\
157-179 \\
404-426 \\
190-212\end{array}$ & $\begin{array}{l}+ \\
+ \\
+ \\
+ \\
+ \\
+ \\
- \\
+\end{array}$ & $\begin{array}{c}0.984 \\
0.95 \\
0.954 \\
0.957 \\
0.954 \\
0.987 \\
0.99 \\
0.954\end{array}$ \\
\hline & & ZBTB7A & $\begin{array}{l}\text { Zinc finger and BTB domain } \\
\text { containing } 7 \mathrm{~A}\end{array}$ & $\begin{array}{l}137-159 \\
154-176 \\
192-214\end{array}$ & $\begin{array}{l}+ \\
+ \\
+\end{array}$ & $\begin{array}{c}0.99 \\
0.986 \\
0.951\end{array}$ \\
\hline & & ZNF219 & $\begin{array}{l}\text { Kruppel-like zinc finger protein } \\
219\end{array}$ & $207-229$ & + & 0.99 \\
\hline ZF07 & $\begin{array}{c}\mathrm{C} 2 \mathrm{H} 2 \text { zinc finger transcription } \\
\text { factors } 7\end{array}$ & ZNF263 & $\begin{array}{c}\text { Zinc finger protein 263, } \\
\text { ZKSCAN12 (zinc finger protein } \\
\text { with KRAB and SCAN domains } \\
\text { 12) }\end{array}$ & $\begin{array}{l}139-153 \\
156-170 \\
210-224 \\
296-310 \\
299-313\end{array}$ & $\begin{array}{l}+ \\
+ \\
+ \\
+ \\
+\end{array}$ & $\begin{array}{l}0.967 \\
0.976 \\
0.967 \\
0.969 \\
0.989\end{array}$ \\
\hline ZF35 & Zinc finger protein ZNF35 & ZNF35 & $\begin{array}{l}\text { Human zinc finger protein } \\
\text { ZNF35 }\end{array}$ & $\begin{array}{c}27-39 \\
550-562\end{array}$ & $\begin{array}{l}- \\
-\end{array}$ & $\begin{array}{l}0.962 \\
0.953\end{array}$ \\
\hline ZF5F & ZF5 POZ domain zinc finger & ZBTB14 & $\begin{array}{l}\text { Zinc finger and BTB domain } \\
\text { containing } 14\end{array}$ & $\begin{array}{c}97-111 \\
133-147\end{array}$ & - & $\begin{array}{l}0.955 \\
0.951\end{array}$ \\
\hline ZTRE & $\begin{array}{c}\text { Zinc transcriptional regulatory } \\
\text { element }\end{array}$ & ZNF658 & Zinc finger protein 658 & $\begin{array}{l}135-151 \\
143-159 \\
152-168 \\
160-176 \\
206-222 \\
214-230\end{array}$ & $\begin{array}{l}- \\
+ \\
- \\
+ \\
- \\
+\end{array}$ & $\begin{array}{c}0.983 \\
0.989 \\
0.984 \\
0.993 \\
0.999 \\
1\end{array}$ \\
\hline
\end{tabular}


Inferring Transcription Factors and microRNAs Associated with Elevated Expression of the Oncogenic B-Cell Lymphoma 11A...

N.N. Laqtom et al.

Table 2. The list of differentially expressed transcription factors in breast cancer tissues compared with control tissue. Three databases for gene expression analysis were used, BioXpress v1.0, Oncomine ${ }^{\mathrm{TM}}$, and Human Protein Atlas. Activators or repressors are included if these factors have shown at least in two databases an increase or decrease in their expression levels, respectively. TFBS and FC indicate transcription factor binding site and fold change.

\begin{tabular}{|c|c|c|c|c|c|c|c|c|c|}
\hline \multirow{2}{*}{$\begin{array}{l}\text { Matrix } \\
\text { Family }\end{array}$} & \multirow{2}{*}{$\begin{array}{l}\text { Detailed Family } \\
\text { Information }\end{array}$} & \multirow{2}{*}{ TF } & \multirow{2}{*}{$\begin{array}{l}\text { Detailed TF } \\
\text { Information }\end{array}$} & \multirow{2}{*}{ ID } & \multirow{2}{*}{ Function } & \multirow{2}{*}{$\begin{array}{c}\text { BioXpress } \\
\text { v1.0 } \\
\text { BC vs. } \\
\text { Normal } \\
\end{array}$} & \multirow{2}{*}{$\begin{array}{c}\text { Oncomine }^{\mathrm{Tm}} \\
\mathrm{BC} \text { vs. Normal }\end{array}$} & \multicolumn{2}{|c|}{$\begin{array}{c}\text { Human Protein } \\
\text { Atlas }\end{array}$} \\
\hline & & & & & & & & Normal & BC \\
\hline BEDF & $\begin{array}{l}\text { BED subclass of } \\
\text { zinc-finger } \\
\text { proteins }\end{array}$ & $\begin{array}{l}\text { ZBED4 } \\
6 \text { TFBSs }\end{array}$ & $\begin{array}{l}\text { Zinc finger, BED-type } \\
\text { containing 4; GC-box } \\
\text { binding sites }\end{array}$ & 9889 & $\begin{array}{l}\text { Transcriptional } \\
\text { activator }\end{array}$ & $\begin{array}{l}\text { Up } 83.19 \% \\
\text { FC: } 0.77\end{array}$ & NA & ++ & $\begin{array}{c}+++ \\
++\end{array}$ \\
\hline \multirow{2}{*}{ EGRF } & \multirow{2}{*}{$\begin{array}{c}\text { EGR/nerve } \\
\text { growth factor } \\
\text { induced protein C } \\
\text { \& related factors }\end{array}$} & $\begin{array}{l}\text { WT1 } \\
\text { 2 TFBSs }\end{array}$ & $\begin{array}{l}\text { Wilms Tumor } \\
\text { Suppressor }\end{array}$ & 7490 & $\begin{array}{c}\text { Transcriptional } \\
\text { activator }\end{array}$ & $\begin{array}{l}\text { Up } 84.96 \% \\
\text { FC: } 3.31\end{array}$ & $\begin{array}{c}\text { Up } \\
\text { P-value: } 0.018 \\
\text { FC: } 1.134\end{array}$ & + & + \\
\hline & & $\begin{array}{c}\text { ZBTB7B } \\
1 \text { TFBS }\end{array}$ & $\begin{array}{c}\text { Zinc finger and BTB } \\
\text { domain containing } 7 \mathrm{~B}\end{array}$ & 51043 & $\begin{array}{l}\text { Transcriptional } \\
\text { activator }\end{array}$ & $\begin{array}{l}\text { Up } 82.3 \% \\
\text { FC: } 0.82\end{array}$ & NA & ++ & $\begin{array}{c}+++ \\
++ \\
+\end{array}$ \\
\hline ZF35 & $\begin{array}{c}\text { Zinc finger } \\
\text { protein ZNF35 }\end{array}$ & $\begin{array}{l}\text { ZNF35 } \\
2 \text { TFBS }\end{array}$ & $\begin{array}{l}\text { Human zinc finger } \\
\text { protein ZNF35 }\end{array}$ & 7584 & $\begin{array}{c}\text { Transcriptional } \\
\text { activator }\end{array}$ & $\begin{array}{l}\text { Up } 73.45 \% \\
\text { FC: } 0.54\end{array}$ & $\begin{array}{c}\text { Up } \\
\text { P-value: } 1.26 \mathrm{E}-4 \\
\text { FC: } 1.327\end{array}$ & ++ & + \\
\hline E2FF & $\begin{array}{c}\text { E2F-myc } \\
\text { activator/cell } \\
\text { cycle regulator }\end{array}$ & $\begin{array}{l}\text { E2F1 } \\
1 \text { TFBS }\end{array}$ & $\begin{array}{l}\text { E2F transcription } \\
\text { factor } 1\end{array}$ & 1869 & $\begin{array}{l}\text { Transcriptional } \\
\text { activator }\end{array}$ & $\begin{array}{l}\text { Up } 97.35 \% \\
\text { FC: } 2.2\end{array}$ & $\begin{array}{l}\text { Insignificant } \\
\text { increase }\end{array}$ & ++ & $\begin{array}{c}+++ \\
++ \\
+ \\
\end{array}$ \\
\hline KLFS & $\begin{array}{l}\text { Kruppel like } \\
\text { transcription } \\
\text { factors }\end{array}$ & $\begin{array}{l}\text { KLF6 } \\
1 \text { TFBS }\end{array}$ & $\begin{array}{l}\text { Core promoter- } \\
\text { binding protein } \\
\text { (CPBP) with } 3 \\
\text { Kruppel-type zinc }\end{array}$ & 1316 & Transcriptionalrepressor & $\begin{array}{c}\text { Down } \\
87.61 \% \\
\text { FC: }-1.43\end{array}$ & $\begin{array}{c}\text { Down } \\
\text { P-value: } 4.20 \mathrm{E}-8 \\
\text { FC: }-3.230\end{array}$ & +++ & $\begin{array}{c}+++ \\
++ \\
+\end{array}$ \\
\hline \multirow{2}{*}{ ZF02 } & \multirow{2}{*}{$\begin{array}{l}\text { C2H2 zinc finger } \\
\text { transcription } \\
\text { factors } 2\end{array}$} & $\begin{array}{l}\text { ZNF300 } \\
8 \text { TFBSS }\end{array}$ & $\begin{array}{l}\text { KRAB-containing zinc } \\
\text { finger protein } 300\end{array}$ & 91975 & Transcriptionalrepressor & $\begin{array}{l}\text { Down } 70.8 \% \\
\text { FC: }-1.57\end{array}$ & $\begin{array}{c}\text { Down } \\
\text { P-value: } 0.033 \\
\text { FC: }-1.069\end{array}$ & +++ & $\begin{array}{c}+++ \\
++\end{array}$ \\
\hline & & $\begin{array}{r}\text { ZNF219 } \\
1 \text { TFBS }\end{array}$ & $\begin{array}{l}\text { Kruppel-like zinc } \\
\text { finger protein } 219\end{array}$ & 51222 & Transcriptionalrepressor & $\begin{array}{c}\text { Down } \\
89.38 \% \\
\text { FC: }-1.55\end{array}$ & $\begin{array}{c}\text { Down } \\
\text { P-value: } 6.29 \mathrm{E}-6 \\
\text { FC: }-1.803\end{array}$ & ++ & $\begin{array}{c}+++ \\
++ \\
+\end{array}$ \\
\hline
\end{tabular}

TFBS: Transcription factor binding site; FC: Fold change

Table 3. The average of differential expression intensities of the transcriptional factors in triple negative breast cancer compared with non-triple negative breast cancer. AV and ARS indicate the average value and the average rank score, respectively.

\begin{tabular}{|c|c|c|c|c|c|c|c|}
\hline \multirow{2}{*}{ TF } & Detailed TF Information & \multicolumn{2}{|c|}{ Non-Triple Negative Breast Cancer } & \multicolumn{2}{|c|}{ Triple Negative Breast Cancer } & \multirow{2}{*}{ Sample Size } & \multirow{2}{*}{ GE0 Profile ID } \\
\cline { 3 - 6 } & ZBED4 & $\begin{array}{c}\text { Zinc finger and BTB domain } \\
\text { containing 7B }\end{array}$ & 303.50 & 67.36 & 344.55 & 71.8 & 19 \\
\hline ZNF35 & Human zinc finger protein ZNF35 & 193.17 & 58.29 & 231.46 & 56.2 & 19 & ID: 77907276 \\
\hline E2F1 & E2F transcription factor 1 & 109.23 & 38.07 & 134.96 & 41.8 & 19 & ID: 77907803 \\
\hline
\end{tabular}

in (Fig.1), there are significant levels of co-regulation between BCL11A and ZBED4 ( $r$ value $=0.196, P$ value $=4.3 \mathrm{E}-28$ ) or $\mathrm{BCL} 11 \mathrm{~A}$ and $\mathrm{E} 2 \mathrm{~F} 1$ ( $r$ value $=0.17, P$ value $=7.84 \mathrm{E}-22$ ) while the expression profile of BCL11A and ZNF35 in breast cancer has shown insignificant correlation. These correlated genes ZBED4 and E2F1with BCL11A are likely to be functionally linked and contribute to regulate a common biological pathway.

\section{High Conservation of ZBED4 and E2F1 Binding Sites between Orthologous Promoters}

In order to examine conservation of transcription factor binding sites between human and mouse, we aligned both species BCL11A promoter sequences ( 600 bp) and found $\sim 56 \%$ of the sequence are not aligned. However, at least a site for ZBED4 and another site for E2F1 are highly conserved between mouse and human (Fig. 2). 
Inferring Transcription Factors and microRNAs Associated with Elevated Expression of the Oncogenic B-Cell Lymphoma 11A... N.N. Laqtom et al.

A
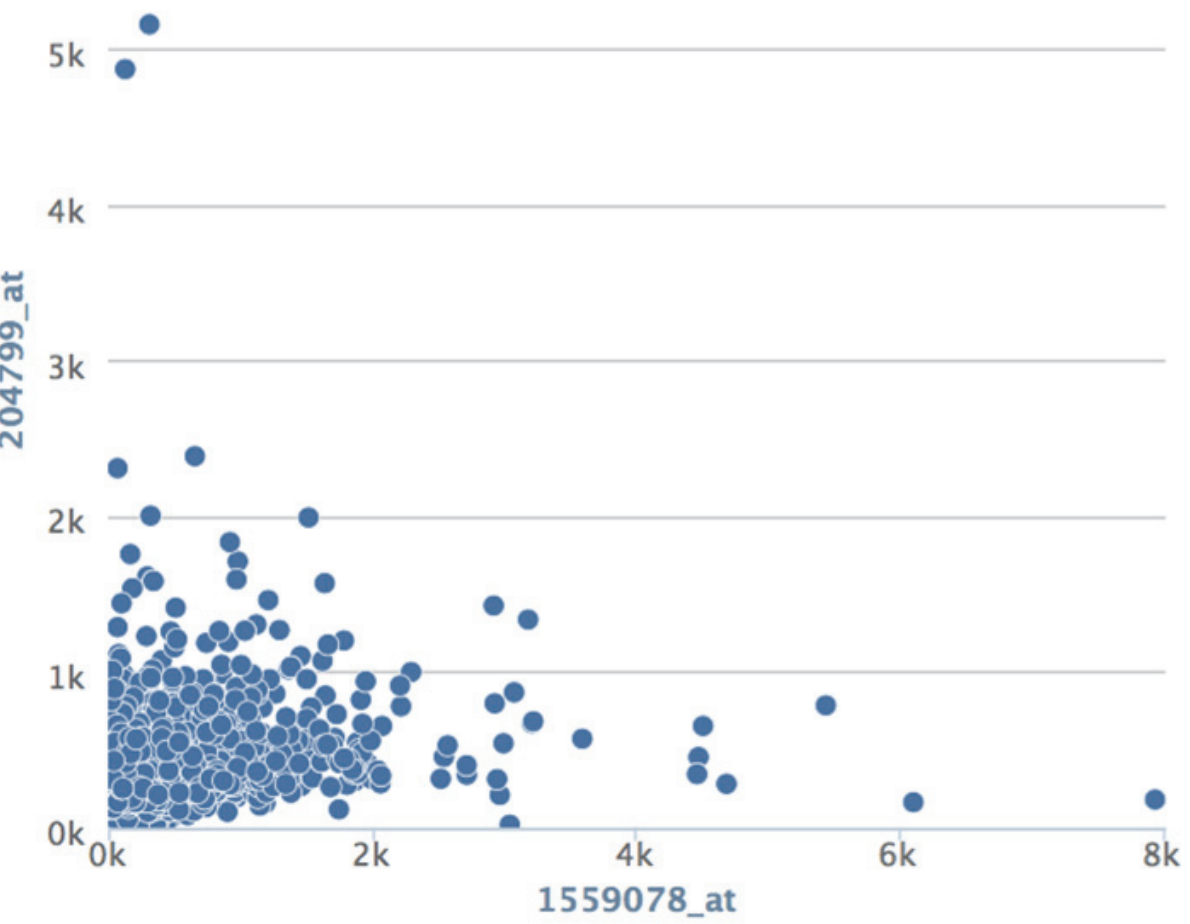

\section{Signal values}

B

$$
2,000
$$

1,500

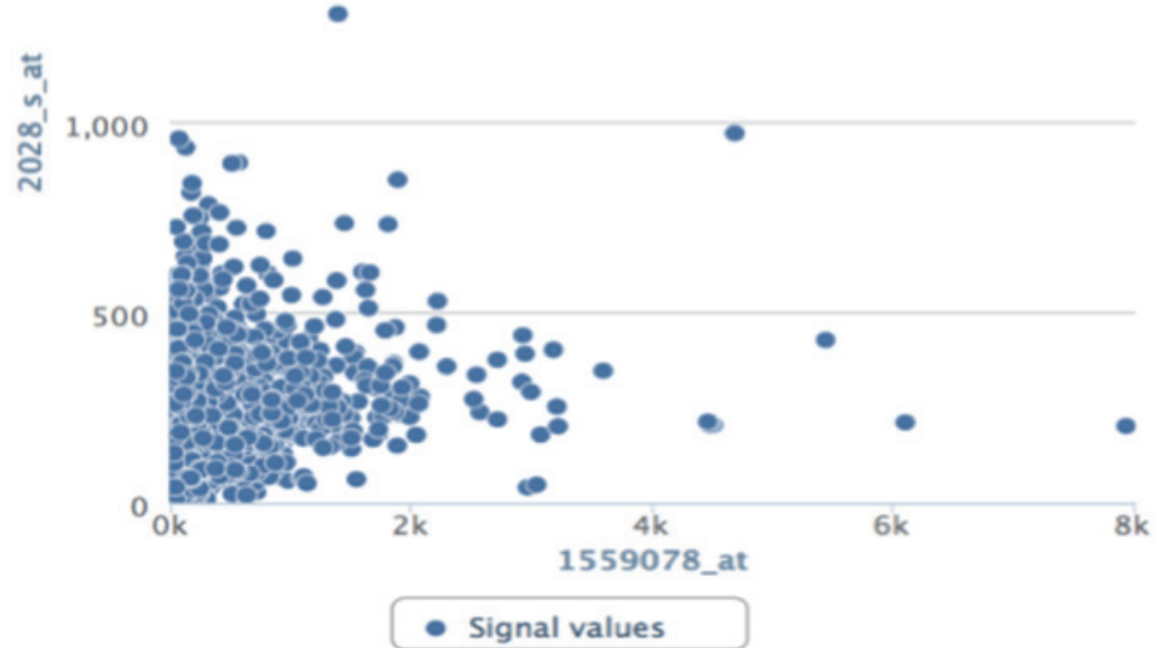

Figure 1. TBCL11A and transcription factors are significantly correlated and co-expressed in breast cancer tissues. The correlation between probe sets for (A) ZBED4 or (B) E2F1 and BCL11A is shown. 


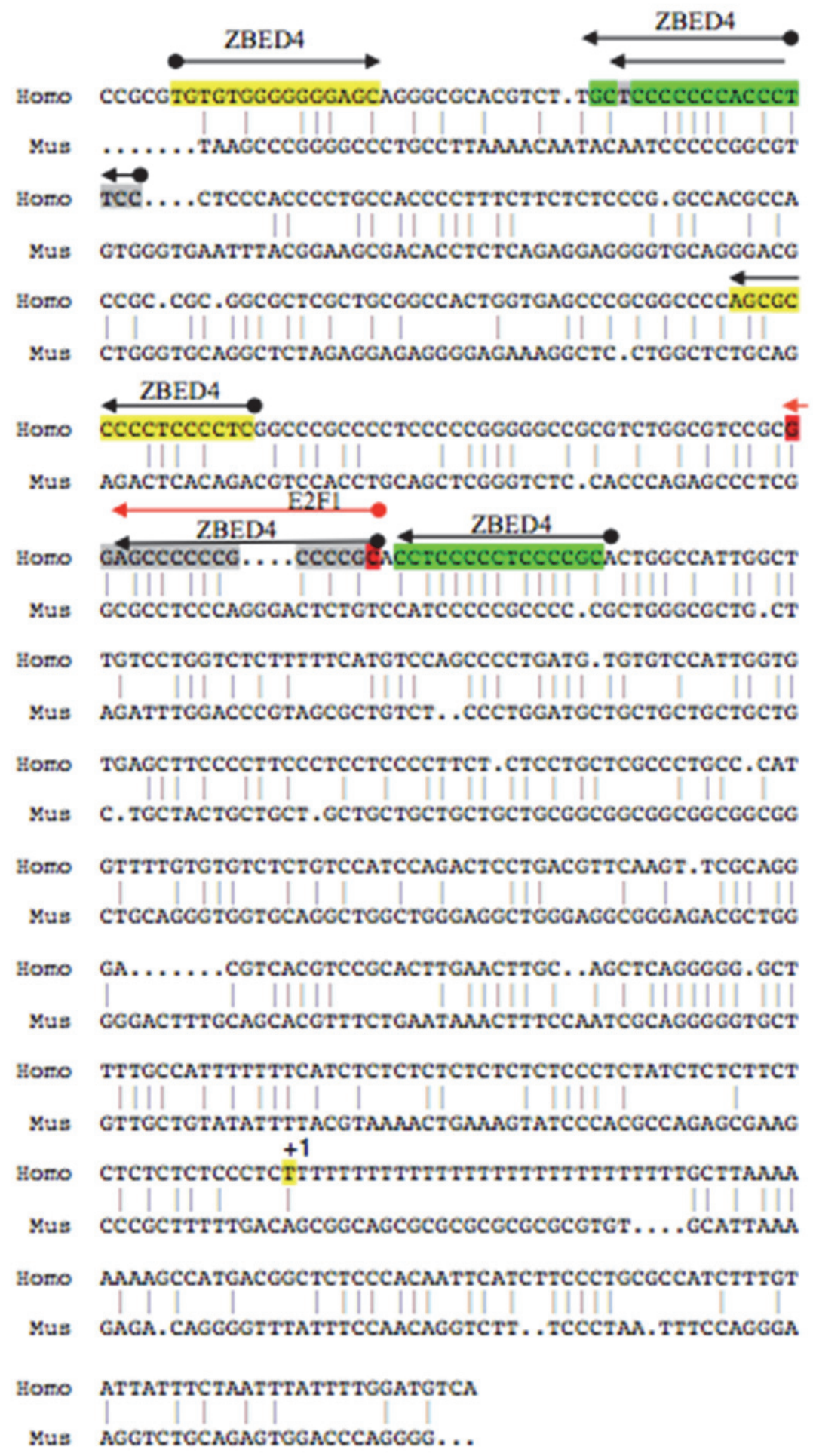

Figure 2. Sequence alignment of BCL11 A promoters showing $44 \%$ conservation between the mouse and human. 


\section{Identification of potential miRNAs related to the high expression of BCL11A in TNBC}

miRNAs can affect gene expression, which target BCL11A mRNAs and suppress its translation into protein. It is possible that the increased level of $B C L 11 \mathrm{~A}$ gene is likely attributed to downregulated miRNAs bearing interaction sequences on the $3^{\prime}$ UTR for BCL11A mRNAs. In order to determine the list of those candidate miRNAs, we obtained the profile of miRNA expression from Triple-Negative Breast Cancer Database (TNBCDb) viewer. The profile compares the non-metastatic or metastatic triple negative breast cancer with normal adjacent breast tissues. Among 108 downregulated miRNAs in TNBC, five of them were identified as able to recognize BCL11A mRNAs by using at least 6-8 complement nucleotide (Table 4). According to TargetScanHuman (Whitehead Institute for Biomedical Research, Cambridge, MA USA), Release 7.1, miR-513a-5p and miR-139-5p have two target sites; whereas, miR-1179, miR-140-5p, and miR-542-3p have one site within BCL11A 3'UTRs.

\section{Discussion}

Breast cancer is the second leading cause of death among females worldwide ${ }^{[1]}$. Genome-wide expression studies helped to determine the heterogeneity and complexity of breast cancer. Breast cancer subtypes: i) ER+/luminal, ii) HER2+ (HER2-enriched), iii) basallike or TNBC, and iv) normal-like have not only distinct expression profiles but also different response patterns to treatments impacting the clinical outcomes ${ }^{[13,14]}$. Among them, TNBC shows the most aggressive biological behavior and highest recurrence rate ${ }^{[15,16]}$. Due to a lack of therapeutic target for TNBC, it remains a significant research challenge. Thus, more understanding for the mechanism underlying the development, maintenance, and metastatic process of TNBC is highly required. Interestingly, increased expression of BCL11A has been reported in B cell lymphoid malignancies as well as breast cancers, in particular triple negative breast cancer. This alternation of $\mathrm{BCL} 11 \mathrm{~A}$ gene expression is related to the cancer pathogenesis ${ }^{[2]}$. Transcription factors and miRNAs are important regulators of gene expression at the transcriptional and post-transcriptional levels, respectively. The promoter region of $B C L 11 A$ remains to be characterized, however, the present work found transcription factors such as KLF6, ZNF300, ZNF219, ZBED4 and E2F1 as potential inducers of BCL11A transcription in cancer cells. Studies on KLF6, ZNF300, ZNF219, ZBED4 and E2F1 revealed that the binding sites of these transcription factors in human promoters can positively affect the BCL11A transcription ${ }^{[17-21]}$. In agreement with the role of BCL11A in breast cancer, KLF6 was reported to have significant effect on breast cancer metastasis and associated with poor clinical outcomes $^{[22]}$. The functions of ZNF300, ZNF219, ZBED4 and E2F1 have not been studied in breast cancer yet, however, the overexpression of ZNF300 resulted in enhanced uncontrolled proliferation and metastasis of HeLa cancer cells in vitro and in vivo ${ }^{[23]}$. Furthermore, E2F1 is suggested to behave as an oncoprotein being an important regulator of cell cycle progression and growth signaling in a tissuespecific manner ${ }^{[24]}$. The higher BCL11A level in triple negative breast cancer tissues correlated with an increase in the expression levels of ZBED4 and E2F1 in this cancer subtype versus others. More validation experiments are essential to confirm whether these transcriptional activators are associated with BCL11A expression in breast cancer and triple negative disease. Besides the transcriptional regulation, $\mathrm{BCL} 11 \mathrm{~A}$ can be regulated post-transcriptionally by miRNAs. We therefore hypothesized a relationship between the

Table 4. A list of downregulated miRNAs in TNBC, which has at least one target site within BCL11A 3'UTRs using Triple-Negative Breast Cancer Database and TargetScanHuman Release 7.1, respectively. FC indicates fold change. ER is estrogen receptor.

\begin{tabular}{|c|c|c|c|}
\hline miRNAs & Control Sample & FC & $\begin{array}{c}\text { Total Potential miRNA } \\
\text { Binding Sites }\end{array}$ \\
\hline \multirow{3}{*}{ miR-139-5p } & Non-TNBC, Her2+,ER+PR+, Normal & -3 & 2 \\
& breast tissue & -3 & \\
\hline miR-513a-5p & Normal adjacent tissue & -2.9 & 2 \\
\hline \multirow{2}{*}{ miR-542-3p } & Matched adjacent normal & -2.69 & 1 \\
\hline miR-1179 & Normal breast tissue & -11.6 & 1 \\
\hline miR-140-5p & Normal breast tissue & -3.8 & 1 \\
\hline BCL11A: B-cell lymphoma 11A & Normal adjacent tissue & -2.15 & \\
\hline
\end{tabular}




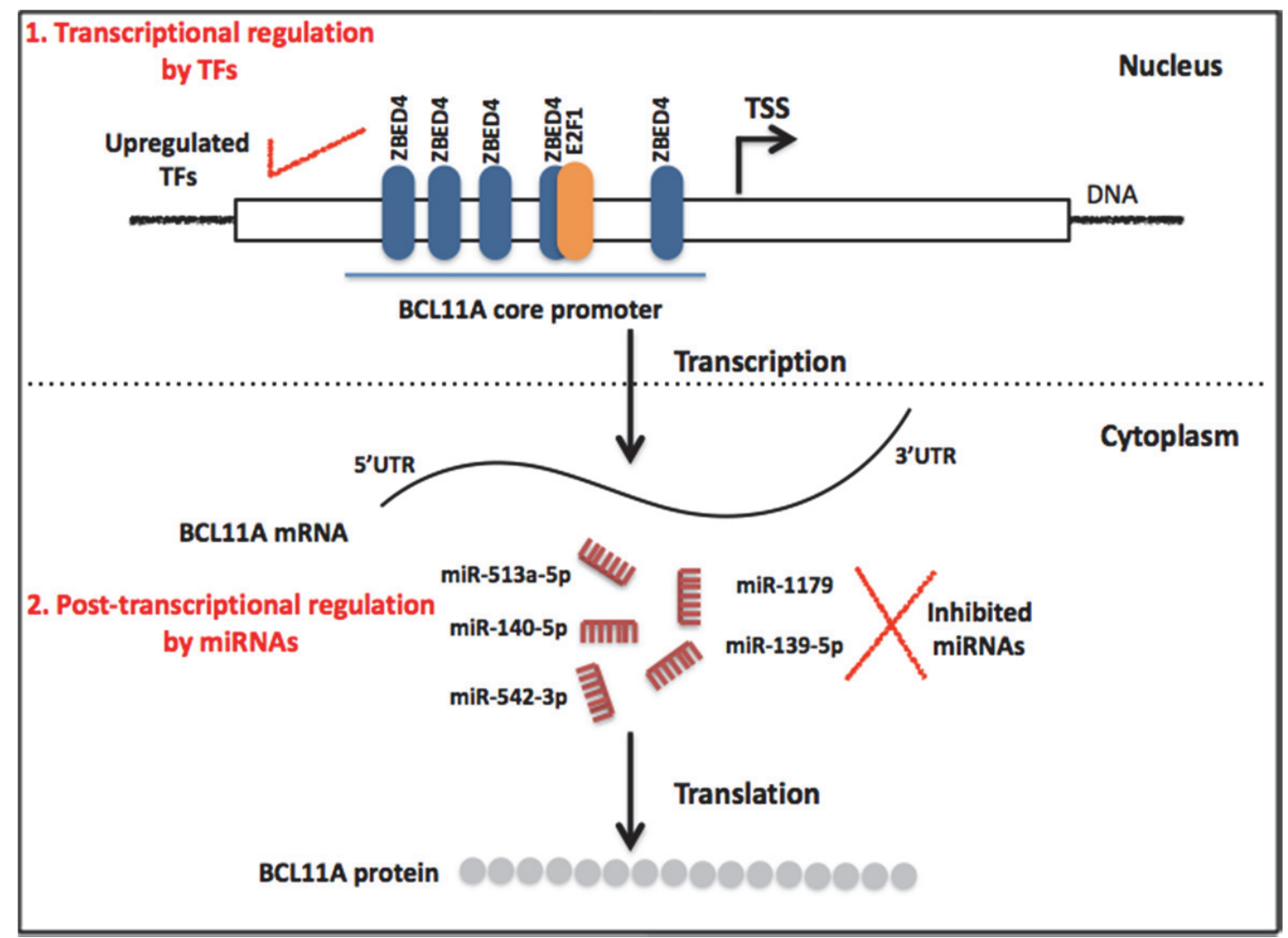

Figure 3. Schematic representation of the suggested mechanism of the regulation of BCL11A expression in triple negative breast cancer. First, the transcriptional regulation is controlled by ZBED4 and E2F1. ZBED4 and E2F1 are known to induce the gene transcription through direct binding to promoter sequences. Second is the post-transcriptional regulation through the inhibition of several miRNAs that are potentially able to interact with BCL11A mRNAs, degrading them and/or blocking their translation. Both levels of regulation can positively influence the expression of BCL11A in triple negative breast cancer cells.

downregulation of miRNAs and increase of BCL11A expression in the triple negative breast cancer. To date, there is no miRNA that has been experimentally validated for targeting BCL11A. Here we predicted that miR-1179, miR-140-5p, miR-542-3p, miR-513a$5 p$ and miR-139-5p, might act as oncosuppressive miRNAs in triple negative breast cancer, through direct targeting of BCL11A. For instance, miR-205 expression downregulation mediates Zinc Finger E-Box Binding Homeobox 1 (ZEB1) and Zinc Finger E-Box Binding Homeobox 2 (ZEB2) gene upregulation. However, the negative regulation in epithelial-mesenchymal transition (EMT) and cancer progression are a consequence of miR-205 targeting ZEB1 and ZEB2 in triple negative breast cancer ${ }^{[25]}$. As another example, increased focal adhesion kinase (FAK) expression is a result of a decrease in miR-7 in tumors compared with normal breast tissue. There is an inverse correlation between miR-7 level and metastasis in human breast cancer tissues ${ }^{[26]}$. Therefore, these data confirm that oncogene expression upregulation can result from the downregulation of specific miRNAs. Taken together, the increased level of BCL11A expression is mediated by upregulation of transcription factors at the transcriptional level and downregulation of miRNAs at the post-transcriptional level in negative breast cancer (Fig. 3).

\section{Reference}

[1] (No author listed) Globocan I. World Heal Organ 2012; Available from <http://globocan.iarc.fr/Pages/fact_sheets_ cancer.aspx>.

[2] Khaled WT, Choon Lee S, Stingl J, Chen X, Raza Ali H, Rueda OM, Hadi F, Wang J, Yu Y, Chin SF, Stratton M, Futreal A, Jenkins NA, Aparicio S, Copeland NG, Watson CJ, Caldas C, Liu P. BCL11A is a triple-negative breast cancer gene with critical functions in stem and progenitor cells. Nat Commun 2015; 6: 5987. 
[3] Satterwhite E, Sonoki T, Willis TG, Harder L, Nowak R, Arriola EL, Liu H, Price HP, Gesk S, Steinemann D, Schlegelberger B, Oscier DG, Siebert R, Tucker PW, Dyer MJS. The BCL11 gene family: involvement of BCL11A in lymphoid malignancies. Blood 2001; 98(12): 3413-3420.

[4] Sankaran VG, Menne TF, Xu J, Akie TE, Lettre G, Van Handel B, Mikkola HK, Hirschhorn JN, Cantor AB, Orkin SH. Human fetal hemoglobin expression is regulated by the developmental stage-specific repressor BCL11A. Science 2008; 322(5909): 1839-1842.

[5] Borg J, Papadopoulos P, Georgitsi M, Gutiérrez L, Grech G, Fanis P, Phylactides M, Verkerk AJMH, van der Spek PJ, Scerri CA, Cassar W, Galdies R, van ljcken W, Ozgür Z, Gillemans N, Hou J, Bugeja M, Grosveld FG, von Lindern M, Felice AE, Patrinos GP, Philipsen S. Haploinsufficiency for the erythroid transcription factor KLF1 causes hereditary persistence of fetal hemoglobin. Nat Genet 2010; 42(9): 801-805.

[6] Hayes J, Peruzzi PP, Lawler S. MicroRNAs in cancer: Biomarkers, functions and therapy. Trends Mol Med 2014; 20(8): 460-469.

[7] Krol J, Loedige I, Filipowicz W. The widespread regulation of microRNA biogenesis, function and decay. Nat Rev Genet 2010;11(9): 597-610.

[8] Cartharius K, Frech K, Grote K, Klocke B, Haltmeier M, Klingenhoff A, Frisch M, Bayerlein M, WernerT. Matlnspector and beyond: promoter analysis based on transcription factor binding sites. Bioinformatics 2005; 21(13): 29332942.

[9] Wan Q, Dingerdissen H, Fan Y, Gulzar N, Pan Y, Wu TJ, Yan C, Zhang H, Mazumder R. BioXpress: an integrated RNA-seqderived gene expression database for pan-cancer analysis. Database (Oxford) 2015; 2015.

[10] Rhodes DR, Yu J, Shanker K, Deshpande N, Varambally R, Ghosh D, Barrette T, Pandey A, Chinnaiyan AM. ONCOMINE: a cancer microarray database and integrated data-mining platform. Neoplasia 2004; 6(1): 1-6.

[11] Uhlén $M$, Björling $E$, Agaton $C$, Szigyarto CA-K, Amini $B$, Andersen $E$, Andersson A-C, Angelidou P, Asplund A, Asplund C, Berglund L, Bergström K, Brumer $H$, Cerjan D, Ekström M, Elobeid A, Eriksson C, Fagerberg L, Falk R, Fall J, Forsberg M, Björklund MG, Gumbel K, Halimi A, Hallin I, Hamsten C, Hansson M, Hedhammar M, Hercules G, Kampf C, Larsson K, Lindskog M, Lodewyckx W, Lund J, Lundeberg J, Magnusson K, Malm E, Nilsson P, Odling J, Oksvold P, Olsson I, Oster E, Ottosson J, Paavilainen L, Persson A, Rimini R, Rockberg J, Runeson M, Sivertsson A, Sköllermo A, Steen J, Stenvall M, Sterky F, Strömberg S, Sundberg M, Tegel $H$, Tourle S, Wahlund E, Waldén A, Wan J, Wernérus $H$, Westberg J, Wester K, Wrethagen U, Xu LL, Hober S, Pontén F. A human protein atlas for normal and cancer tissues based on antibody proteomics. Mol Cell Proteomics 2005; 4(12): 1920-1932.

[12] Raju R, Paul AM, Asokachandran V, George B, Radhamony L, Vinaykumar M, Giriiadevi R, Pill MR. The Triple-Negative
Breast Cancer Database: an omics platform for reference, integration and analysis of triple-negative breast cancer data. Breast Cancer Res 2014; 16(6): 490.

[13] Perou CM, Sørlie T, Eisen MB, van de Rijn M, Jeffrey SS, Rees CA, Pollack JR, Ross DT, Johnsen H, Akslen LA, Fluge O, Pergamenschikov A, Williams C, Zhu SX, Lønning PE, Børresen-Dale AL, Brown PO, Botstein D. Molecular portraits of human breast tumours. Nature 2000; 406(6797): 747-752.

[14] Sørlie T, Perou CM, Tibshirani R, Aas T, Geisler S, Johnsen $H$, Hastie $T$, Eisen MB, van de Rijn M, Jeffrey SS, Thorsen T, Quist H, Matese JC, Brown PO, Botstein D, Lønning PE, Børresen-Dale AL. Gene expression patterns of breast carcinomas distinguish tumor subclasses with clinical implications. Proc Natl Acad Sci U S A 2001; 98(19): 1086910874.

[15] OvcaricekT, Frkovic SG, Matos E, Mozina B, Borstnar S. Triple negative breast cancer - prognostic factors and survival. Radiol Oncol 2011; 45(1): 46-52.

[16] Dent R, Trudeau M, Pritchard KI, Hanna WM, Kahn HK, Sawka CA, Lickley LA, Rawlinson E, Sun P, Narod SA. Triple-negative breast cancer: clinical features and patterns of recurrence. Clin Cancer Res 2007; 13(15 Pt 1): 4429-4434.

[17] Guo H, Lin Y, Zhang H, Liu J, Zhang N, Li Y, Kong D, Tang Q, Ma D. Tissue factor pathway inhibitor-2 was repressed by $\mathrm{CpG}$ hypermethylation through inhibition of KLF6 binding in highly invasive breast cancer cells. BMC Mol Biol 2007; 8: 110.

[18] Qiu H, Xue L, Gao L, Shao H, Wang D, Guo M, Li W. Identification of the DNA binding element of the human ZNF300 protein. Cell Mol Biol Lett 2008; 13(3): 391-403.

[19] Takigawa Y, Hata K, Muramatsu S, Amano K, Ono K, Wakabayashi M, Matsuda A, Takada K, Nishimura R, Yoneda T. The transcription factor Znf219 regulates chondrocyte differentiation by assembling a transcription factory with Sox9. J Cell Sci 2010; 123 (Pt 21): 3780-3788.

[20] Mokhonov W, Theendakara VP, Gribanova YE, Ahmedli NB, Farber DB. Sequence-specific binding of recombinant Zbed4 to DNA: insights into Zbed4 participation in gene transcription and its association with other proteins. PLOS One 2012; 7(5): e35317.

[21] Fry CJ, Pearson A, Malinowski E, Bartley SM, Greenblatt J, Farnhamt PJ. Activation of the murine dihydrofolate reductase promoter by E2F1: a requirement for CBP recruitment. J Biol Chem 1999; 274(22): 15883-15891.

[22] Hatami R, Sieuwerts AM, Izadmehr S, Yao Z, Qiao RF, Papa L, Look MP, Smid M, Ohlssen J, Levine AC, Germain D, Burstein D, Kirschenbaum A, DiFeo A, Foekens JA, Narla G. KLF6-SV1 drives breast cancer metastasis and is associated with poor survival. Sci Transl Med 2013; 5(169): 169ra12.

[23] Wang T, Wang XG, Xu JH, Wu, XP, Qiu, HL, Yi H, Li WX. Overexpression of the human ZNF300 gene enhances growth and metastasis of cancer cells through activating NF-kB pathway. J Cell Mol Med 2012; 16(5): 1134-1145. 
Inferring Transcription Factors and microRNAs Associated with Elevated Expression of the Oncogenic B-Cell Lymphoma 11A... N.N. Laqtom et al.

[24] Eymin B, Gazzeri S, Brambilla C, Brambilla E. Distinct pattern of E2F1 expression in human lung tumours: E2F1 is upregulated in small cell lung carcinoma. Oncogene 2001;20(14): 1678-1687.

[25] Piovan C, Palmieri D, Di Leva G, Braccioli L, Casalini P, Nuovo G, Tortoreto M, Sasso M, Plantamura I, Triulzi T, Taccioli C, Tagliabue E, lorio MV, Croce CM. Oncosuppressive role of p53-induced miR-205 in triple negative breast cancer. Mol Oncol 2012; 6(4): 458-472.

[26] Kong X, Li G, Yuan Y, He Y, Wu X, Zhang W, Wu Z, Chen T, Wu W, Lobie PE, Zhu T. MicroRNA-7 inhibits epithelial-tomesenchymal transition and metastasis of breast cancer cells via targeting FAK expression. PLoS One 2012; 7(8): e41523. 


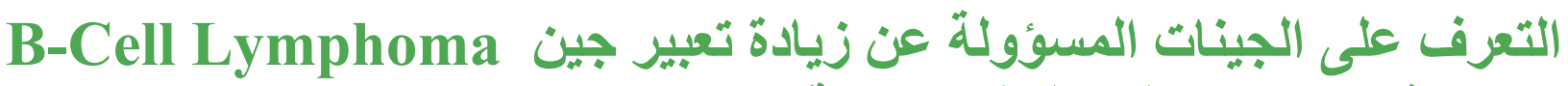 11A في سرطان الثي ثلاثي السلبية}

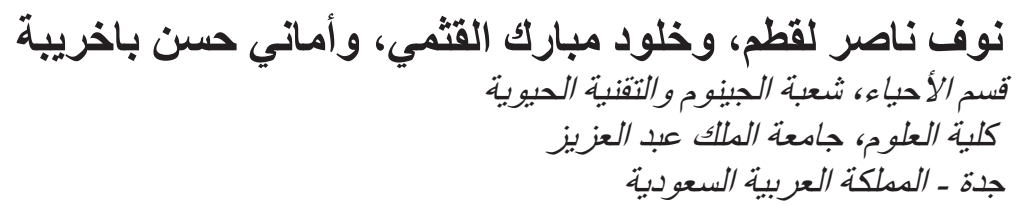

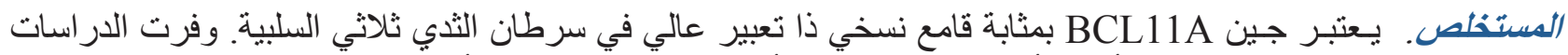



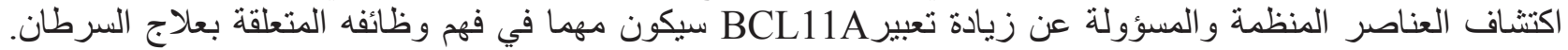
تعتبر بروتينات النسخ (Transcription factors وجزيئات (microRNAs) من الهم العناصر العناصة المنظمة للتعبير الجيني.

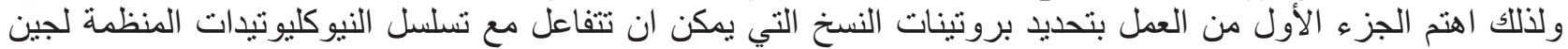
BCL11A

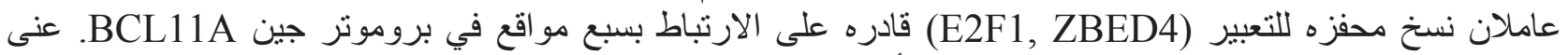

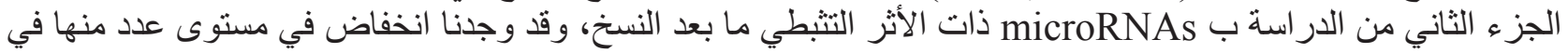
خلايا سرطان الثذي ثلاثي السلبية مثل التل و التي لديها القدرة على الارتباط الجزيئي بموقع او اكثر مع BCL11A mRNAs معطلة عمليه الترجمة للبروتين. استخدام

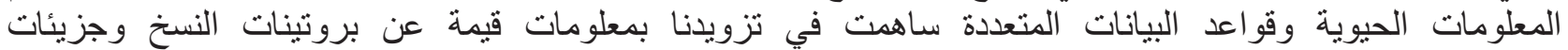
microRNAs 